\title{
Red-Shift Index Concept in Solvent Effects of Chromophore-Substituted Metallophthalocyanines: A Look at the Empirical Relationship of the Macroscopic Properties of the Solute-Solvent Interactions
}

\author{
Victor Akpe $\cdot$ Abimbola Ogunsipe $\cdot$ Christian Madu $\cdot$ Hjalmar Brismar
}

Received: 26 April 2014/ Accepted: 30 November 2014/Published online: 22 February 2015

(C) Springer Science+Business Media New York 2015

\begin{abstract}
Solvent effects on the UV/vis spectra of metallopthalocyanines (MPcs) have been interpreted using the red-shift index concept $\left(R_{s} I\right)$. The concept connects empirically, direct, experimental, easily accessible optical spectral data, which are explained by considering the differential behavior of the solute-solvent interactions at the ground state and excited state using the spectral values of MPcs along with the derived concept, called the associated solvation energy (ASE). $R_{s} I$ is formulated from three fundamental parameters, which are: ground state electronic absorption spectrum, polarization red-shift and a scaling factor of MPc $\left(N_{\text {dye }}\right)$ in the respective solvents. The $R_{s} I$ is a reflection of the index value of the chromophore substituent of MPc in the solvent; thus, the concept can be used as a solvatochromic parameter to study a wide range of supramolecular and heterocyclic compounds that can be modified at their periphery or 'handles'. Particularly, in this study, the concept has been used to rank MPc candidates by using the statistical mean performance of the solvatochromic parameters, which are red shift index, polarizability efficiency and ASE. We hereby review the solvent effects on the UV/vis spectra of substituted and unsubstituted MPcs.
\end{abstract}

Keywords Aggregation behavior - Solvation - Polarization red-shift - Solvent polarity · Metallophthalocyanine

\section{Introduction}

Metallophthalocyanines (MPcs) are classical organometallic dyes and many of their derivatives exhibit intrinsic properties that make them suitable in optical data storage [1],

\footnotetext{
V. Akpe $(\bowtie) \cdot$ H. Brismar

Department of Applied Physics, Royal Institute of Technology, 10691 Stockholm, Sweden e-mail: victorakpe@gmail.com
}

\author{
A. Ogunsipe \\ School of Science and Technology, National Open University of Nigeria, Lagos, Nigeria \\ C. Madu \\ Department of Chemistry, Collin College, Preston Ridge Campus, Frisco, TX 75035, USA
}


chemical sensors [2], non-linear optics [3], and in light-driven applications [4-6]. In solution, MPcs display an intense blue-green color, with extinction coefficients greater than $10^{5} \mathrm{~L} \cdot \mathrm{mol}^{-1} \cdot \mathrm{cm}^{-1}$. Structurally, they have a well-ordered macrocyclic backbone that contains the fusion of four isoindole units conjugated to four azomethine nitrogen atoms to give stable $18 \pi$-electron configuration structures. MPcs have two distinct bands; the Q-band as it is so called, that appears in the visible region $(600-700 \mathrm{~nm})$, which is the focus of our investigation, and the B (Soret) band in the UV region (300-350 nm). The Q-band is the result of HOMO-LUMO transitions within the MPc molecule [7], while the transition that gives the B-band is from a level lower than the HOMO.

Addition of chromophore-substituents (CS) to the MPcs ring (peripherally or nonperipherally) and metallation have been reported not only to increase the distance between the planar macrocycle rings but also to improve the solubility or solvation process [8-11], which have an overall effect on the chemical and physical properties [12].

Solvent-effects, in a broad sense, have been explained by several investigators [12-15], specifically with the purpose of addressing the complexity of chemical phenomena in solution. Commonly this has necessitated the development of models, application of computational techniques, and sometimes of mathematical concepts, to explain observations about the chemical system of interest. To this end, the research theme that is common to all in this vast topic is: "the quest to understand the behavior of molecules in their environments". The aim of this work is therefore to explain holistically, solvent-effects on the behaviors of CS-MPcs at the ground and excited states using the red-shift index $\left(R_{s} I\right)$. This concept provides the platform for the formulation of a mathematical operation that is not only reproducible but also based on good empirical deductions from experimental spectral values of MPcs in the respective solvents.

\section{Solvent Effects on UV/Vis Spectra of MPcs}

In the ground state, the solute's charge separation is expected to be small because electrons are held tightly, causing the solute-solvent electrostatic interactions to be minimal. At the excited state, on the other hand, electrons are less tightly held, making the solute's charge separation and solute-solvent interactions increase because the electrons are more polarizable, resulting in redshifts during the solvation process. According to the Franck-Condon principle, vertical transitions should alter the solute's electron charge distribution without affecting its internal geometry, so that the overall effect should not affect the spectra. Thus the direction of solute's spectral shift upon solvation depends on the relative polarity of the solute in the ground and the excited states, the solvent's polarity and the polarizability as discussed in a review of the chemistry [16].

Solvent effects on UV/vis spectra of MPc have been explained based on the macroscopic properties, described in this work as the nature of solvent (e.g. polar, non-polar, coordinating solvent, the polarization red-shift of the dye in response to the solvent's polarity) and the nature of the CS in MPcs.

\subsection{Polarization Red-Shift of MPcs}

A significant change in solvent polarity can cause a corresponding shift in spectral wavelength. Polarization red-shifts of MPcs do not correlate well with dipole moments, as shown by recent studies $[17,18]$. Thus, in this work, we have deliberately not included dipole moment functions in the study because the polarization red-shift correlates well with other parameters listed in the Tables 1,2 and 3. 


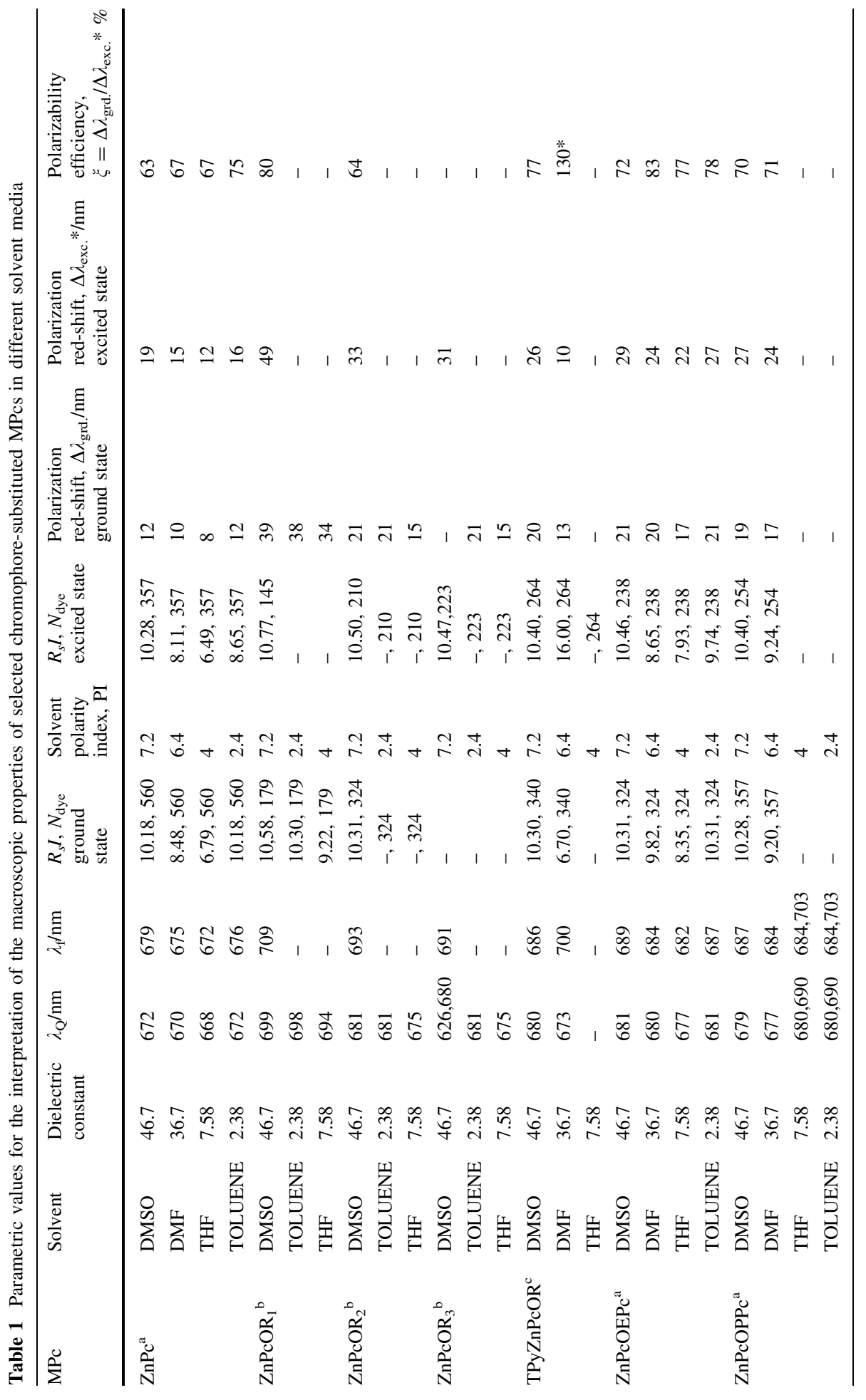




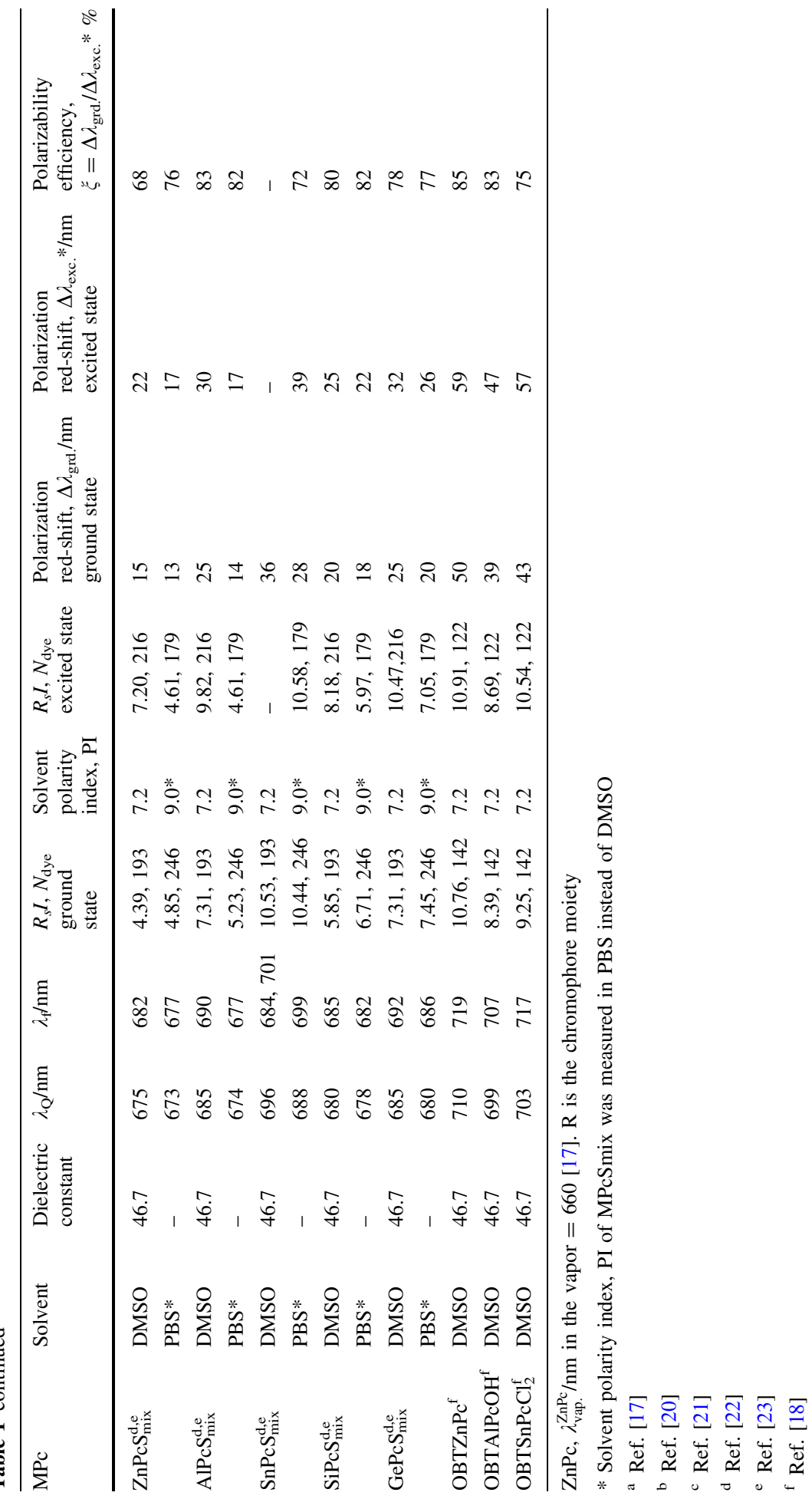




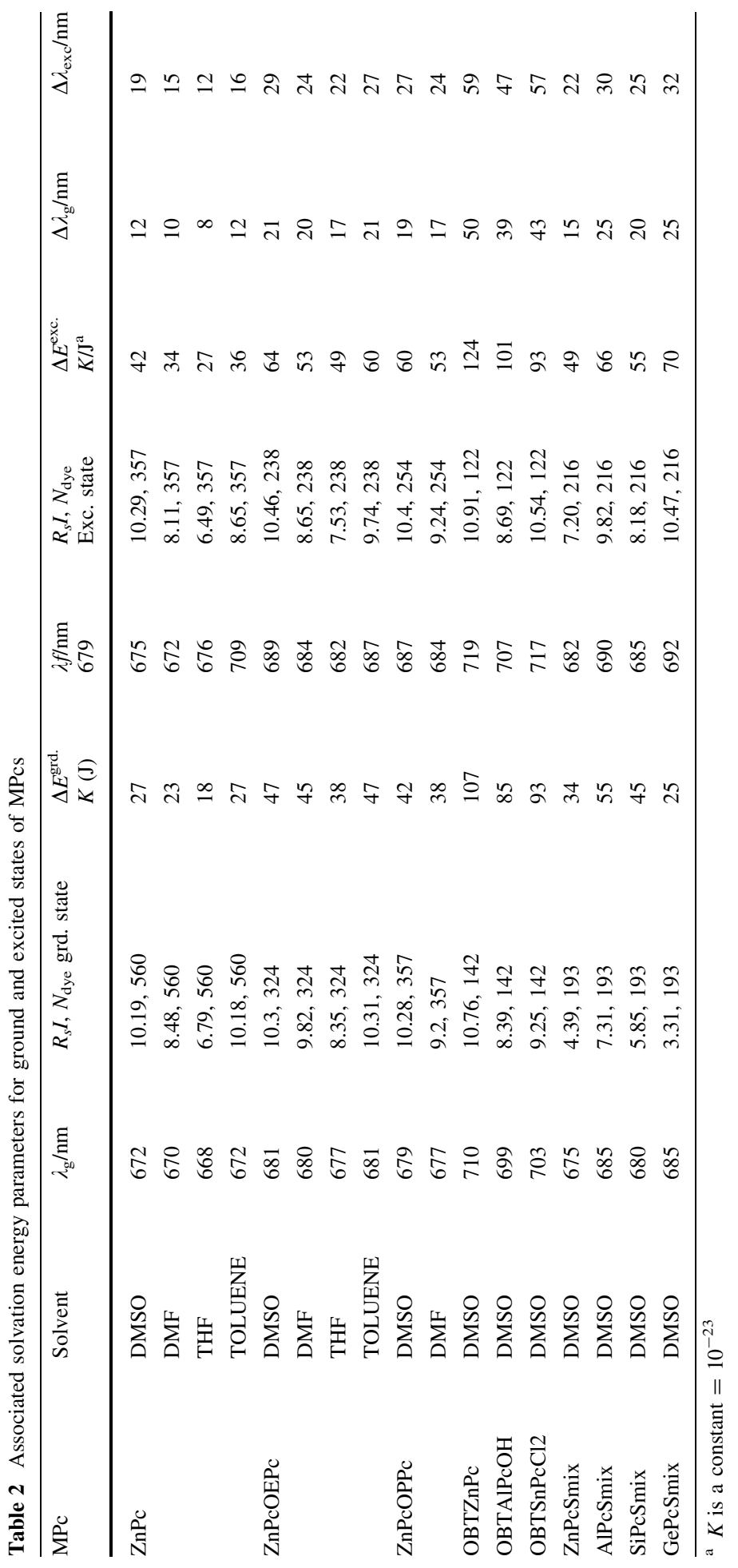


Table 3 CS-MPc rank based on the statistical mean performance of the solvatochromic parameters

\begin{tabular}{|c|c|c|c|c|c|c|}
\hline \multirow[t]{2}{*}{ MPc } & \multicolumn{3}{|c|}{ Polarizability } & \multirow{2}{*}{$\begin{array}{l}\text { Mean } \\
\text { performance }\end{array}$} & \multirow{2}{*}{$\begin{array}{l}\text { Standard } \\
\text { deviation }\end{array}$} & \multirow[b]{2}{*}{ Rank } \\
\hline & $R_{s} I$ & Efficiency & ASE & & & \\
\hline TPyZnPcOR(DMF) & 7 & 130 & 31 & 56 & 65.2 & 5 \\
\hline OBTZnPc(DMSO) & 11 & 85 & 109 & 68.3 & 51.1 & 1 \\
\hline ZnPcOEPc(DMF) & 10 & 83 & 45 & 46 & 36.5 & 8 \\
\hline AlPcSmix(DMSO) & 7 & 83 & 53 & 47.7 & 38.3 & 6 \\
\hline OBTAlPcOH(DMSO) & 8 & 83 & 81 & 57.3 & 42.7 & 4 \\
\hline $\operatorname{AlPcSmix}(\mathrm{PBS})$ & 5 & 82 & 30 & 39 & 39.3 & 16 \\
\hline $\operatorname{SiPcSmix}(\mathrm{PBS})$ & 7 & 82 & 42 & 44.7 & 37.5 & 12 \\
\hline ZnPcOR(DMSO) & 11 & 80 & 88 & 59.7 & 42.3 & 2 \\
\hline SiPcSmix(DMSO) & 6 & 80 & 46 & 44 & 37 & 11 \\
\hline $\mathrm{ZnPcOEPc}$ (toluene) & 10 & 78 & 45 & 44.3 & 34 & 10 \\
\hline GePcSmix(DMSO) & 7 & 78 & 53 & 46 & 36 & 8 \\
\hline ZnPcSmix(PBS) & 5 & 76 & 30 & 37 & 36 & 19 \\
\hline $\mathrm{ZnPc}$ (toluene) & 10 & 75 & 27 & 37.3 & 33.7 & 18 \\
\hline OBTSnPcCl2(DMSO) & 9 & 75 & 90 & 58 & 43.1 & 3 \\
\hline $\mathrm{ZnPcOEPc}(\mathrm{DMSO})$ & 10 & 75 & 45 & 43.3 & 32.5 & 13 \\
\hline $\operatorname{SnPcSmix}(\mathrm{PBS})$ & 10 & 72 & 59 & 47 & 32.7 & 7 \\
\hline ZnPcOPPc(DMF) & 9 & 71 & 37 & 39 & 31 & 16 \\
\hline ZnPcOPPc(DMSO) & 10 & 70 & 41 & 40.3 & 30 & 14 \\
\hline ZnPcSmix(DMSO) & 4 & 68 & 31 & 34.3 & 32.1 & 20 \\
\hline $\mathrm{ZnPc}(\mathrm{DMF})$ & 8 & 67 & 21 & 32 & 31 & 22 \\
\hline $\mathrm{ZnPc}(\mathrm{THF})$ & 7 & 67 & 19 & 31 & 31.7 & 23 \\
\hline ZnPcOR2(DMSO) & 10 & 64 & 45 & 39.7 & 27.4 & 15 \\
\hline $\mathrm{ZnPc}(\mathrm{DMSO})$ & 10 & 63 & 27 & 33.3 & 27.1 & 21 \\
\hline
\end{tabular}

We look at two case studies of CS-MPcs based on the studies by Baylis and coworkers [13, 14]: (1) those that are mostly influenced by a momentary transition dipole during the optical absorption process in the ground state electronic absorption spectra, and (2) those that are influenced by a transition from the ground state to the excited state. For CS-MPc in the ground state, absorption spectra in non-polar solvents with bathochromic (red) shifts, we have used these defined parameters: $(1)$ the Q-band $\left(\lambda_{\mathrm{Qmax}}\right)$ of the unsubstituted MPc in the vapor phase, where we have referenced the experimental Q-band of unsubstituted $\mathrm{ZnPc}$ in the vapor phase [17]; (2) the Q-band of the unsubstituted MPc in solution.

The polarization red-shift is therefore described as the spectral difference between the Q-band of CS-MPc in solution and the Q-band of the unsubstituted ZnPc in the vapor phase, which is the reference value. The red-shift on the other hand is the difference between the Q-band of CS- MPc and unsubstituted MPc in the same solvent. Because the solubility of both substituted and unsubstituted MPcs may differ widely in the same solvent, we use the wavelength of the Q-band of $\mathrm{ZnPc}$ in the vapor phase as reference. 
2.2 Polarization Red-Shift of MPcs with Respect to Spectral Wavelengths

\subsubsection{Case 1: Polarization Red-Shift at the Ground State}

By applying the work of Baylis and co-workers [13, 14], where they qualitatively described the polarization effect of a solute in a solvent, we can deduce that the polarization red-shift of a CS-MPc in solution $\left(\Delta \lambda_{\text {soln. }}^{\mathrm{MPc} \text { (subst.) }}\right)$ is the spectral wavelength difference between the substituted MPc (at the Q-band) $\left(=\lambda_{\mathrm{Qmax} \text { soln. }}^{\mathrm{MP} \text { (subst) }}\right)$ in the solvent and the unsubstituted $\mathrm{ZnPc}$ (at the $\mathrm{Q}$-band, $=\lambda_{\mathrm{Qmax} v a p \text {. }}^{\mathrm{ZnPc} \text { (unsubt) }}$ in the vapor phase):

$$
\Delta \lambda_{\text {soln. }}^{\mathrm{MPc} \text { (subst.) }}=\lambda_{\mathrm{Qmax} \text { soln. }}^{\mathrm{MPc} \text { (subst.) }}-\lambda_{\mathrm{Qmax} \text { vap. }}^{\mathrm{ZnPc} \text { (unsubst.) }} .
$$

The momentary transition dipole of substituted MPc, the red-shift, $\Delta \lambda^{* \text { subst. }}$, is the spectral wavelength difference between the Q-band of substituted MPc and the Q-band of unsubstituted MPc dissolved in the same solvent:

$$
\Delta \lambda^{* \text { subst. }}=\lambda_{\mathrm{Qmax} \text { soln. }}^{\mathrm{MPc} \text { (subst. }}-\lambda_{\mathrm{Qmax} \text { soln. }}^{\mathrm{MPc} \text { (unsubst. }} .
$$

On the other hand, the momentary transition dipole of unsubstituted MPc induced by solvent, denoted as $\Delta \lambda^{* \text { unsubst. }}$, is the spectral wavelength difference of Q-band of unsubstituted MPc in the solvent it is dissolved in and the Q-band of unsubstituted $\mathrm{ZnPc}$ in the vapor phase:

$$
\Delta \lambda^{* \text { unsubst. }}=\lambda_{\mathrm{Qmax} \text { soln. }}^{\mathrm{MPc} \text { (unsubst.) }}-\lambda_{\mathrm{Qmax} \text { vap. }}^{\mathrm{ZnP} \text { (unsubs.) }}
$$

Addition of Eqs. 2 and 3 gives:

$$
\begin{gathered}
\Delta \lambda^{* \text { subst. }}+\Delta \lambda^{* \text { unsubst. }}=\lambda_{\mathrm{Qmax} \text { soln. }}^{\mathrm{MPc} \text { (subst.) }}-\lambda_{\mathrm{Qmax} \text { vap. }}^{\mathrm{ZnPc} \text { (unsubst.) }} \\
\Delta \lambda^{* \text { subst. }}+\Delta \lambda^{* \text { unsubst. }}=\Delta \lambda^{\text {subst. }}
\end{gathered}
$$

From Eq. $4 b$, the polarization red-shift of a CS-MPc $(\Delta \lambda)$ ' is the sum of momentary transition dipoles of both the substituted chromophore in the MPc ring $\left(\Delta \lambda^{* \text { subst. }}\right)$ and the unsubstituted $\mathrm{ZnPc}$ ring in the vapor phase.

\subsubsection{Case 2: Polarization Red-Shift at the Excited State}

For a CS-MPc transition, from the ground state to the excited state, we consider these factors: (1) the difference between the permanent dipole moment (that is, between the ground and the excited states of the solute) and (2) the effect of the Franck-Condon principle, grouped in this work as the observed Stokes shift effect $\left(\Delta_{\text {Stokes }}\right)$, with the assumption that hydrogen bonds are negligible or absent.

Therefore, the polarization red-shift of a CS-MPc transition at the excited state is described as the sum of the red-shift and Stokes' shift observed.

$$
\begin{gathered}
\Delta \lambda^{\mathrm{exc}}=\Delta \lambda^{* \text { subst. }}+\Delta_{\text {Stokes soln }}^{\mathrm{MPc} \text { subst. }} \\
\Delta \lambda^{\mathrm{exc}}=\left(\lambda_{\text {Qmax soln. }}^{\text {MPc subst. }}-\lambda_{\text {Qmax soln. }}^{\mathrm{MPc} \text { unsubst. }}\right)+\left(\lambda_{f \text { soln. }}^{\text {MPc subst. }}-\lambda_{\text {Qmax soln. }}^{\mathrm{MPc} \text { subst. }}\right)
\end{gathered}
$$




$$
\Delta \lambda^{\mathrm{exc}}=\lambda_{f \text { soln. }}^{\mathrm{MPc} \text { subst. }}-\lambda_{\mathrm{Qmax} \text { soln. }}^{\mathrm{MPc} \text { unsubt. }}
$$

where

$$
\Delta_{\text {Stokes }}^{\mathrm{MPc} \text { subst. }}=\lambda_{f \text { soln. }}^{\mathrm{MPc} \text { subst. }}-\lambda_{\mathrm{Qmax} \text { soln. }}^{\mathrm{MPc} \text { subt. }} .
$$

Since the solubility of an unsubstitituted MPc and its derivative, CS-MPc may differ, the experimental value of $\mathrm{ZnPc}$ determined in the vapor phase is applied.

Therefore the polarization red-shift of an MPc at the excited state from Eq. $5 \mathrm{c}$ is replaced with:

$$
\Delta \lambda^{\mathrm{exc}}=\lambda_{f \text { soln. }}^{\mathrm{MPc} \text { subst. }}-\lambda_{\mathrm{vap}}^{\mathrm{ZnPc}}
$$

where $\lambda_{f \text { soln. }}^{\mathrm{MPc} \text { (subst.) }}$ is the fluorescence emission wavelength of the substituted MPc in solution.

The polarizability efficiency of an MPc transition from the ground state to the excited state is defined in this study as the ratio of the polarization red-shift of the ground state to the polarization red-shift at the excited state:

Polarizability efficiency, $\xi$, of an MPc is given by:

$$
\xi=\frac{\Delta \lambda_{\mathrm{grd}}^{\mathrm{MPc}}}{\Delta \lambda_{\mathrm{exc}}^{\mathrm{MPc}}}
$$

\subsection{Nature of Chromophore Substituents of MPcs}

The type of CS introduced into an MPc ring, either peripherally or axially, can result in significant UV/vis spectral shifts, hypsochromic or bathochromic. These CSs, along with their canonical resonance structures, can influence the magnitude and the direction of the shift. It is expected, for instance, that, the more canonical resonance structures or the higher is the extended conjugation of a CS, the higher should be the shift. Since only electron donating CSs were investigated in this paper, the derived equations, used to describe the fundamental parameters, are within the defined range.

\section{Red-Shift Index and Associated Solvation Energy}

\subsection{Derivation of the Red-Shift Index $\left(R_{s} I\right)$ and Associated Solvation Energy (ASE) at the Ground State}

When solvent molecules associate with a solute during the solvation process, energy is either released or absorbed. However, an electronically excited solute is expected to carry with it a quantum of energy, which we term "associated solvation energy" (ASE) in this work. Since some investigators have shown that the spectral approach can be used to predict solute-solvent behavior [11, 19], we show in this paper the relationship of ASE with the Q-band of MPc transitions and how they are related to RsI.

It is expected that the ASE of a CS-MPc should be higher than the unsubstituted MPc in the vapor phase. This is because in the vapor phase, the unsubstituted MPc molecules are not charged and there are no CSs that can aid the solvation process to produce stable species in the vapor phase. In line with this argument, the ASE of a solute-solvent 
interaction with a polarization red-shift in the ground state along the spectral approach can be described as follows:

Considering Eq. 4a, the ASE of an MPc transition that has a frequency $(v)$ at the ground state can be written as:

$$
\Delta E_{\text {solv. }}^{\mathrm{grd}}=h v_{\mathrm{vap} .}^{\mathrm{ZnPc}}-h v_{\text {soln. }}^{\mathrm{MPc}}
$$

where $\Delta E$ is given as the associated energy of solvation, $h$ is Planck's constant, $v$ is frequency and the speed of light $c=v \lambda$.

From Eq. 7a,

$$
\frac{\Delta E_{\text {solv. }}^{\mathrm{grd} .}}{h v_{\text {vap. }}^{\mathrm{ZnPc}}}=\frac{\left(h v_{\text {vap. }}^{\mathrm{ZnPc}}-h v_{\text {soln. }}^{\mathrm{MPc}}\right)}{h v_{\text {vap. }}^{\mathrm{ZnPc}}}=\frac{\left(v_{\text {vap. }}^{\mathrm{ZnPc}}-v_{\text {soln. }}^{\mathrm{MPc}}\right)}{v_{\text {vap. }}^{\mathrm{ZnPc}}}=1-\frac{v_{\text {soln. }}^{\mathrm{MPc}}}{v_{\text {vap. }}^{\mathrm{ZnPc}}}
$$

Since the wavelength is inversely proportional to the frequency, Eq. $7 \mathrm{~b}$ becomes:

$$
\frac{\Delta E_{\text {solv. }}^{\mathrm{grd} .} \lambda_{\mathrm{vap} .}^{\mathrm{ZnPc}}}{h c}=\frac{\lambda_{\text {soln. }}^{\mathrm{MPc}}-\lambda_{\mathrm{vap} .}^{\mathrm{ZnPc}}}{\lambda_{\mathrm{soln} .}^{\mathrm{MPc}}}=1-\frac{\lambda_{\text {vap. }}^{\mathrm{ZnPc}}}{\lambda_{\text {soln. }}^{\mathrm{MPc}}}
$$

Therefore,

$$
\frac{\Delta E_{\mathrm{solv} .}^{\mathrm{grd} .} \lambda_{\mathrm{soln} .}^{\mathrm{MPc}}}{h c}=\frac{\Delta \lambda_{\mathrm{soln}}^{\mathrm{MPc}}}{\lambda_{\mathrm{vap} .}^{\mathrm{ZnP}}}
$$

Multiplying Eq. $7 \mathrm{~d}$ by $N_{\text {dye }}$, the scaling factor of the dye, gives:

$$
\frac{\Delta E_{\text {solv. }}^{\mathrm{grd} .} \lambda_{\mathrm{soln} .}^{\mathrm{MPc}} \times N_{\text {dye }}}{h c}=\frac{\Delta \lambda_{\mathrm{soln} .}^{\mathrm{MPc}} \times N_{\text {dye }}}{\lambda_{\text {vap. }}^{\mathrm{ZnP}}}
$$

From Eq. 7e, on the right hand side of the equation, the red-shift index, $R_{S} I$, is therefore defined as the ratio of the polarization red-shift of the CS-MPc (sample) to the absorption maximum peak wavelength of the unsubstituted ZnPc in the vapor phase [17] multiplied by a scaling factor, $N_{\text {dye }}$.

$$
R_{s} I=\frac{\Delta \lambda_{\text {soln. }}^{\text {sample }} \times N_{\text {dye }}}{\lambda_{\text {max vap. }}^{\text {ref }}}
$$

where,

$$
N_{\text {dye }}^{\text {grd. }}=\frac{10 \lambda_{\text {max soln. }}^{\text {sample }}}{\Delta \lambda_{\text {soln. }}^{\text {sample }}}=10 \frac{\lambda_{\text {max soln. }}^{\text {sample }}}{\lambda_{\text {max soln. }}^{\text {sample }}-\lambda_{\text {max vap. }}^{\text {reference }}}
$$

$\lambda_{\text {max vap. }}^{\text {reference }}$ is the wavelength of the Q-band of $\mathrm{ZnPc}$ in the vapor phase. $\Delta \lambda_{\text {soln. }}^{\text {sample }}$ is the polarization red-shift of the investigated sample, that is the CS-MPc in solution. $N_{\text {dye }}[18$, 19] is the scaling factor of the dye when dissolved in a given solvent. It is determined by selecting the solvent with the highest solvent polarity index value. The scaling factor, $N_{\text {dye }}$, is measured from three parameters: the polarization red-shift of the chromophore-substituted MPc, wavelength of the unsubstituted $\mathrm{ZnPc}$ at the Q-band in the vapor phase, and the choice of solvent with the highest polarity index value (PI) that the solute (MPc) is most soluble in. 
Similarly from Eq. 7e, the ASE of an MPc at the ground state, $\Delta E_{\text {solv. }}^{\text {grd. }}$, is defined as:

$$
\Delta E_{\mathrm{solv} .}^{\mathrm{grd} .}=\frac{R_{s} I \times h c}{\lambda_{\mathrm{soln} .}^{\mathrm{MPc}} \times N_{\mathrm{dye}}}
$$

where the units for the following physical constants are: $h=6.626 \times 10^{-34} \mathrm{~m}^{2} \cdot \mathrm{kg} \cdot \mathrm{s}^{-1}$, $c=3 \times 10^{8} \mathrm{~m} \cdot \mathrm{s}^{-1}$, and $\lambda=\mathrm{nm}\left(=10^{-9} \mathrm{~m}\right)$, making the dimensions of $\Delta E$ in Joules.

3.2 Derivation of the Red-Shift Index $\left(R_{S} I\right)$ and Associated Solvation Energy at the Excited State

Equation 5e describes the polarization red-shift of an MPc at the excited state. Therefore ASE for an MPc transition with a frequency $v$ at the excited state is given by:

$$
\Delta E_{\text {solv. }}^{\mathrm{exc}}=h v_{\mathrm{vap} .}^{\mathrm{ZnPc}}-h v_{\mathrm{f}}^{\mathrm{MPc}}
$$

If all the previous steps from Eqs. $7 b-8$ are followed stepwise then:

$$
\begin{gathered}
\frac{\Delta E_{\text {solv. }}^{\text {exc }}}{h v_{\text {vap. }}^{\mathrm{ZnPc}}}=\frac{h v_{\text {vap. }}^{\mathrm{ZnPc}}-h v_{\mathrm{f}}^{\mathrm{MPc}}}{h v_{\text {vap. }}^{\mathrm{ZnPc}}}=\frac{v_{\text {vap. }}^{\mathrm{ZnPc}}-v_{\mathrm{f}}^{\mathrm{MPc}}}{v_{\text {vap. }}^{\mathrm{ZnPc}}}=1-\frac{v_{\mathrm{f}}^{\mathrm{MPc}}}{v_{\text {vap. }}^{\mathrm{ZnPc}}} \\
\frac{\Delta E^{\mathrm{exc}} \lambda_{\text {vap. }}^{\mathrm{ZnPc}}}{h c}=\frac{\lambda_{\mathrm{f}}^{\mathrm{MPc}}-\lambda_{\text {vap. }}^{\mathrm{ZnPc}}}{\lambda_{\text {vap. }}^{\mathrm{MPc}}}
\end{gathered}
$$

where $\lambda_{\mathrm{f} \mathrm{soln} .}^{\mathrm{MPc}}-\lambda_{\mathrm{vap} .}^{\mathrm{ZnP}}$ is the polarization red-shift at the excited state, $\Delta \lambda^{\mathrm{exc}}$,

$$
\frac{\Delta E^{\mathrm{exc}} \lambda^{\mathrm{MPc}}}{h c}=\frac{\Delta \lambda^{\mathrm{exc}}}{\lambda_{\mathrm{vap} .}^{\mathrm{ZnPc}}}
$$

Multplying Eq. 12a all through by $N_{\text {dye }}$, gives:

$$
N_{\text {dye }} \frac{\Delta E^{\mathrm{exc}} \lambda_{\mathrm{vap}}^{\mathrm{MPc}}}{h c}=N_{\text {dye }} \frac{\Delta \lambda^{\mathrm{exc}}}{\lambda_{\text {vap. }}^{\mathrm{ZnPc}}}=R_{s} I^{\mathrm{exc}}
$$

The $R_{s} I$ and $N_{\text {dye }}$ at the excited states are therefore:

$$
R_{s} I^{\text {exc }}=\frac{\Delta \lambda_{\text {soln. }}^{\text {exc }} \times N_{\text {dye }}}{\lambda_{\text {vap. }}^{\mathrm{ZnPc}}}
$$

where

$$
N_{\text {dye }}^{\text {exc }}=\frac{10 \lambda_{\mathrm{f}}^{\mathrm{MPc}}}{\Delta \lambda^{\mathrm{exc}}}=10 \frac{\lambda_{\mathrm{f}}^{\mathrm{MPc}}}{\lambda_{\mathrm{f}}^{\mathrm{MPc}}-\lambda_{\mathrm{vap}}^{\mathrm{ZnP}}}
$$

From Eq. 12a:

$$
\Delta E_{\mathrm{solv} .}^{\mathrm{exc}}=\frac{\Delta \lambda^{\mathrm{MPcexc}} \times h c}{\lambda_{\mathrm{f}}^{\mathrm{MPc}}} .
$$




\section{Discussion and Results}

Table 1 shows eleven parametric values used to study the solvent effects of fifteen MPcs. It also contains a list of common solvents. These parameters are: the Q-band $\left(\lambda_{\max }\right)$ at the ground electronic state, fluorescence emission wavelength $\left(\lambda_{\mathrm{f}}\right)$, the polarization-red shifts at the ground and excited states $\left(\Delta \lambda^{\text {grd }}\right.$ and $\left.\Delta \lambda^{\text {exc }}\right)$, red-shift index at the ground and excited states $\left(R_{s} I\right), N_{\text {dye }}$ at the ground and excited states, polarizability efficiency, and solvent parameters: solvent polarity index $(P I)$ and dielectric constant, $F(\mathrm{MPc})$, which is a constant for a specific MPc; solvent donor number $(D N)$ and refractive index of solvents have not been included in this table but their correlation with the Q-band and the polarization redshift have been discussed previously $[17,18]$.

The CS behavior of MPc should change in the different solvent environments [17-20]. The magnitude of the change depends on the nature of solvent, polarity index of solvent, the dielectric value of solvent and the polarity of the CS of MPc during the solvation process either in the ground state absorption or the excited state. Figure 1a shows the scatter plot for the variation of the $R_{S} I$ values of the CS in the respective solvents, where the $R_{S} I$ values vary in different solvents. The observed change is due mainly to the interaction between the functional groups present in CS and the solvent. The more polar the solvent, the higher the $R_{S} I$ value, except in toluene, where the $R_{s} I$ shows remarkably high values for $\mathrm{ZnPc}$ (10.18), $\mathrm{ZnPcOR}_{1}$ (10.30), and $\mathrm{ZnPcOEPc}$ (10.31), which are attributed to the induced positive effects of the methyl group of the toluene ring and CS-MPc during the solvation process in the ground state and are observed to fall during the solvation process at the excited state. Figure $1 \mathrm{~b}$ also shows the scatter plot of the variation of $N_{\text {dye }}$ of the different CS-MPc in the same solvent (DMSO). The plot shows that different CS-MPcs, in the same solvent, have different $N_{\text {dye }}$ s and that the $N_{\text {dye }}$ of the ground state is also distinguishable from the one in the excited state. Thus, the $N_{\text {dye }}$ values make it possible to measure a wide range of $R_{s} I$ values of CS-MPcs, either in the ground or excited states, because it takes into cognizance the solute-solvent interactions and, without the multiplication by the scaling factor $N_{\text {dye, }}$ the overlapped $R_{s} I$ values will not be distinguishable. It can therefore be adduced succinctly that the $N_{\text {dye }}$ is the 'acid-test' of the $R_{s} I$.

Figure 1c shows the chart diagram of the comparative study of the solvent polarity index with the $R_{s} I$ values at the ground and excited states, from which these two classes of behaviors were observed: (1) $R_{S} I$ at the excited state is greater than $R_{S} I$ of the ground state as compared with polarity index (PI) of solvent, and (2), where the $R_{s} I$ of the excited state is lower than the $R_{s} I$ at the ground state as compared with the PI of solvents; the categories are shown below:

\begin{tabular}{ll}
\hline Positive solvent effect & Negative solvent effect \\
\hline ZnPc(DMSO) & ZnPc(DMF) \\
ZnPcOEPc(DMSO) & $\mathrm{ZnPc}(\mathrm{THF})$ \\
OBTZnPc(DMSO) & $\mathrm{ZnPc}$ (toluene) \\
OBTAlPcOH(DMSO) & ZnPcOEPc(DMF) \\
OBTSnPcCl $($ DMSO) & ZnPcOEPc(toluene) \\
GePcSmix(DMSO) & AlPcSmix(PBS) \\
AlPcSmix(DMSO) & SiPcSmix(PBS) \\
ZnPcSmix(DMSO) & GePcSmix(PBS) \\
SiPcSmix(DMSO) & ZnPcSmix(PBS) \\
\hline
\end{tabular}



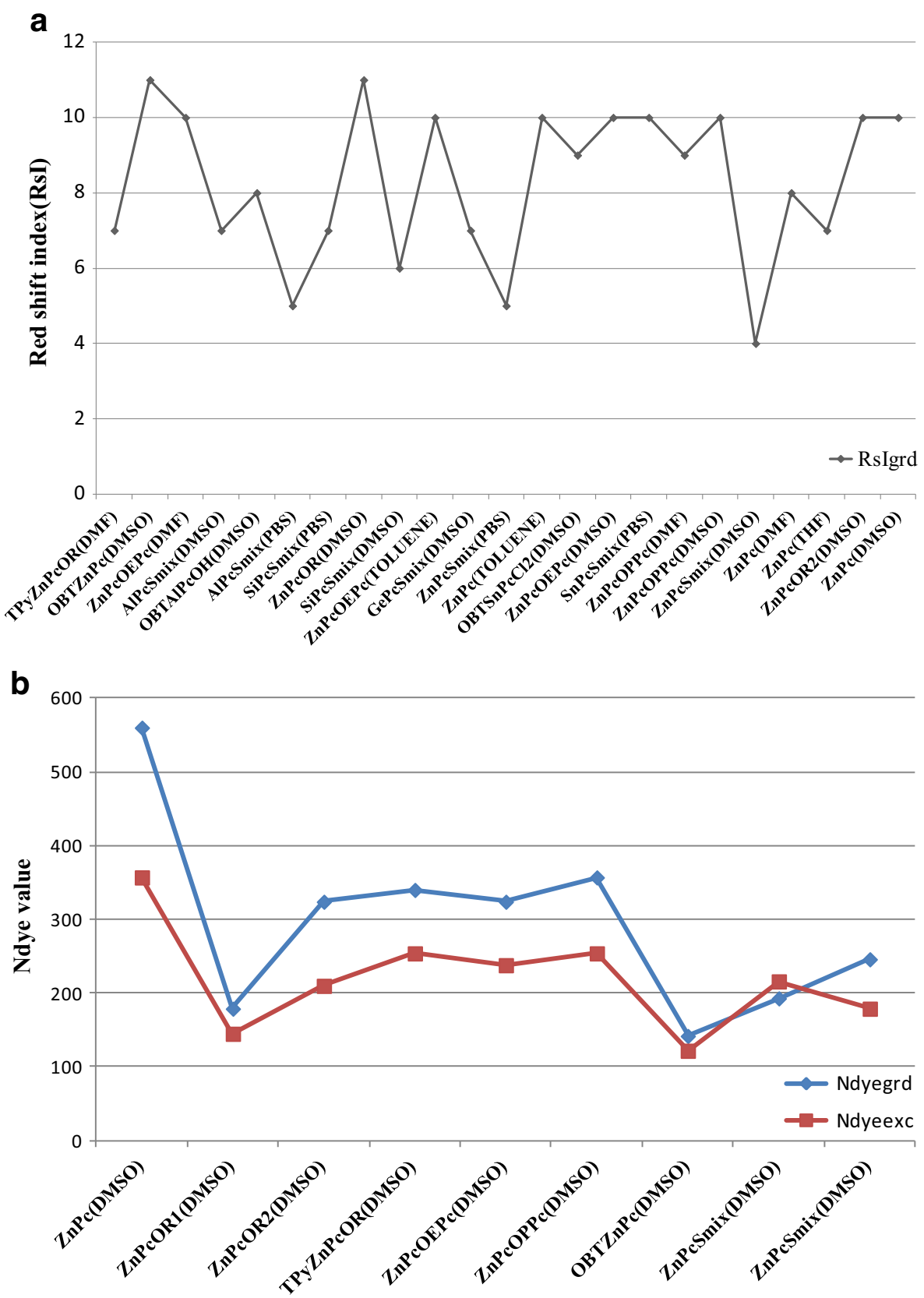

Fig. 1 a Red-shift index variation at the ground state. b Relative $\mathrm{N}_{\text {dye }}$ of MPc as the CS varies for both the ground and excited states. c Polarity index of solvents compared with $R_{s} I:(\mathbf{d}-\mathrm{i})$ positive solvent effect illustration in solute-solvent interactions (d-ii). Negative solvent effect illustration in solute-solvent interactions 

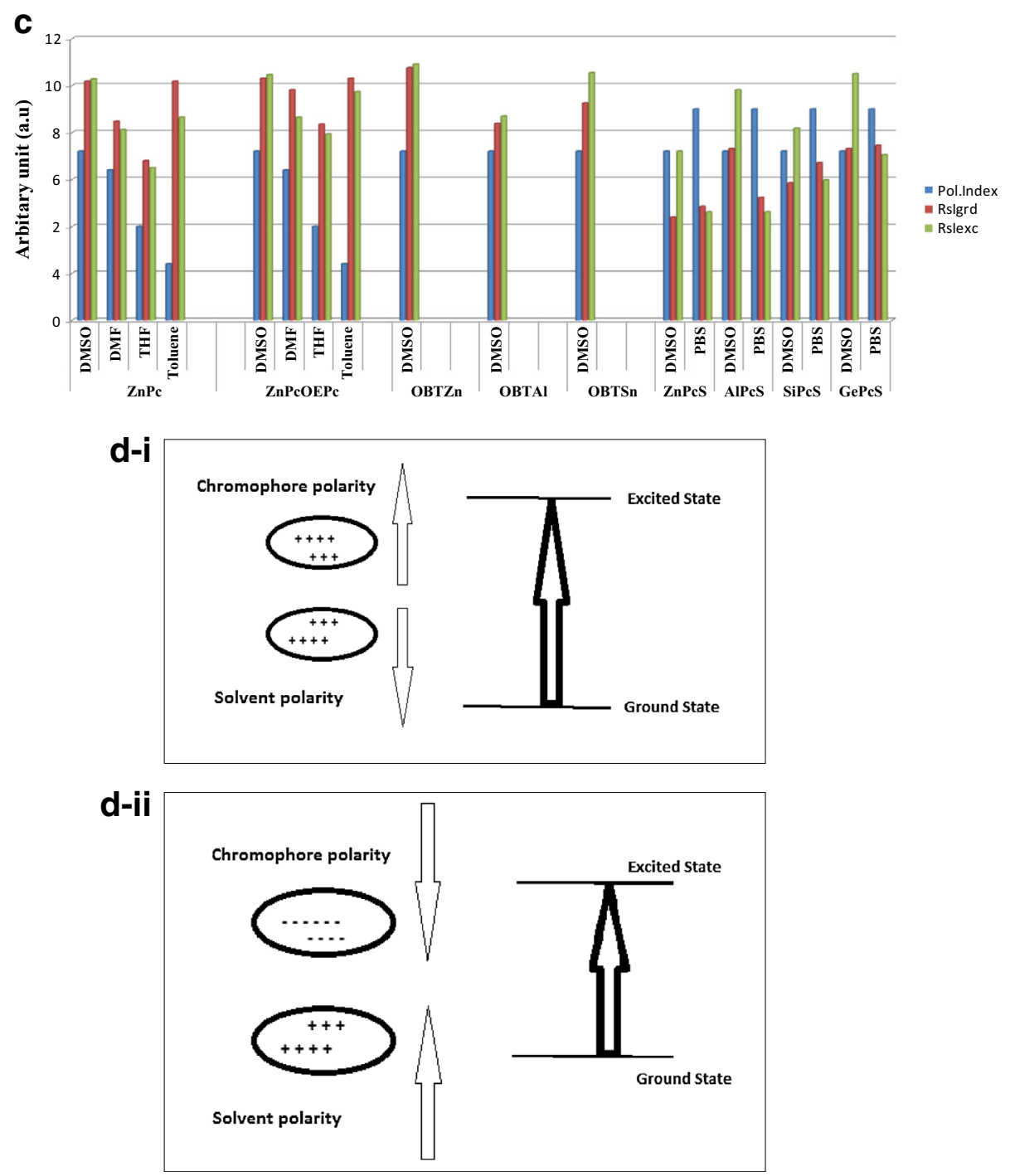

Fig. 1 continued

Figures 1d (i) and (ii) show the explanation of both the positive and negative solvent effects. When the chromophore moiety induces a positive inductive effect into a polar aprotic environment such as DMSO with two methyl groups (induced positive effect in the environment), the result is an induced electrostatic charge separation of the solute-solvent interactions causing the distance between the planar macrocycle rings of the CS-MPc to increase during the solvation process to bring about the increased red-shift observed for the positive solvent effect. Negative solvent effect on the other hand results from a situation where the interaction between the chromophore moiety from the MPc and the solvent environment brings about a decrease in the electrostatic charge separation followed by an 

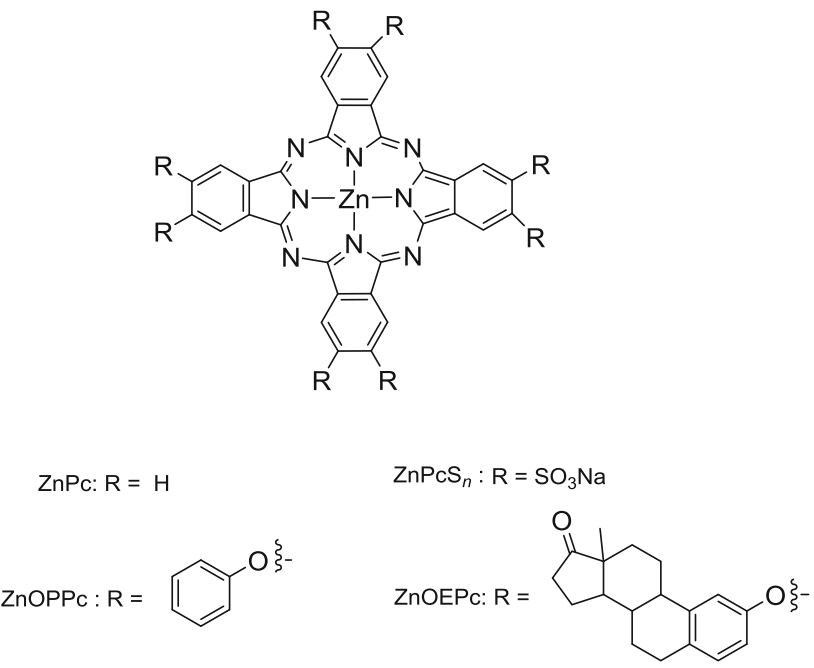

Scheme 1 Representative molecular structures of $\mathrm{ZnPc}, \mathrm{ZnPcS}_{n}$, ZnOPPc, and $\mathrm{ZnOEPc}$

accompanied decrease of the planar macrocycle rings of the CS-MPc during the solvation process to bring about the decreased red-shift and the decrease in the red shift index value calculated as the CS-MPc moves from the ground state to the excited state (Scheme 1).

Regarding the substitution of electron-donating moieties into MPc rings, the nature of the central metal ion in the MPc has been reported to enhance the solvation process [12]. It is thus expected that the addition of these groups should increase the following parameters: red-shift values in solution, polarization red-shift values and subsequently the $R_{s} I$ values as shown in Fig. 2 (where the polarization red-shift was compared with polarizability efficiency), and Fig. 1c (where the red-shift index compared with the polarity index of solvents). However, certain solvents are also known to improve the solvation process such as the coordinating effect of DMF, which we observed in our investigation to induce the solvent medium to give rise to the negative solvent-effect seen for TPyZnPcOR (DMF) in Fig. 2, marked by an unusually high polarizability efficiency because of a higher solvation

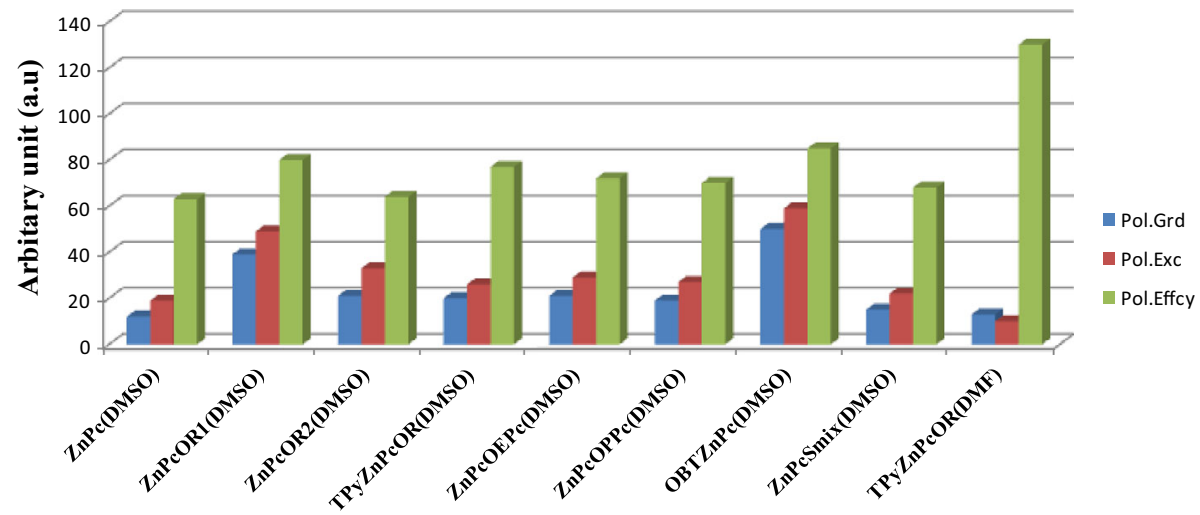

Fig. 2 Polarizability efficiency compared with polarization red shift 

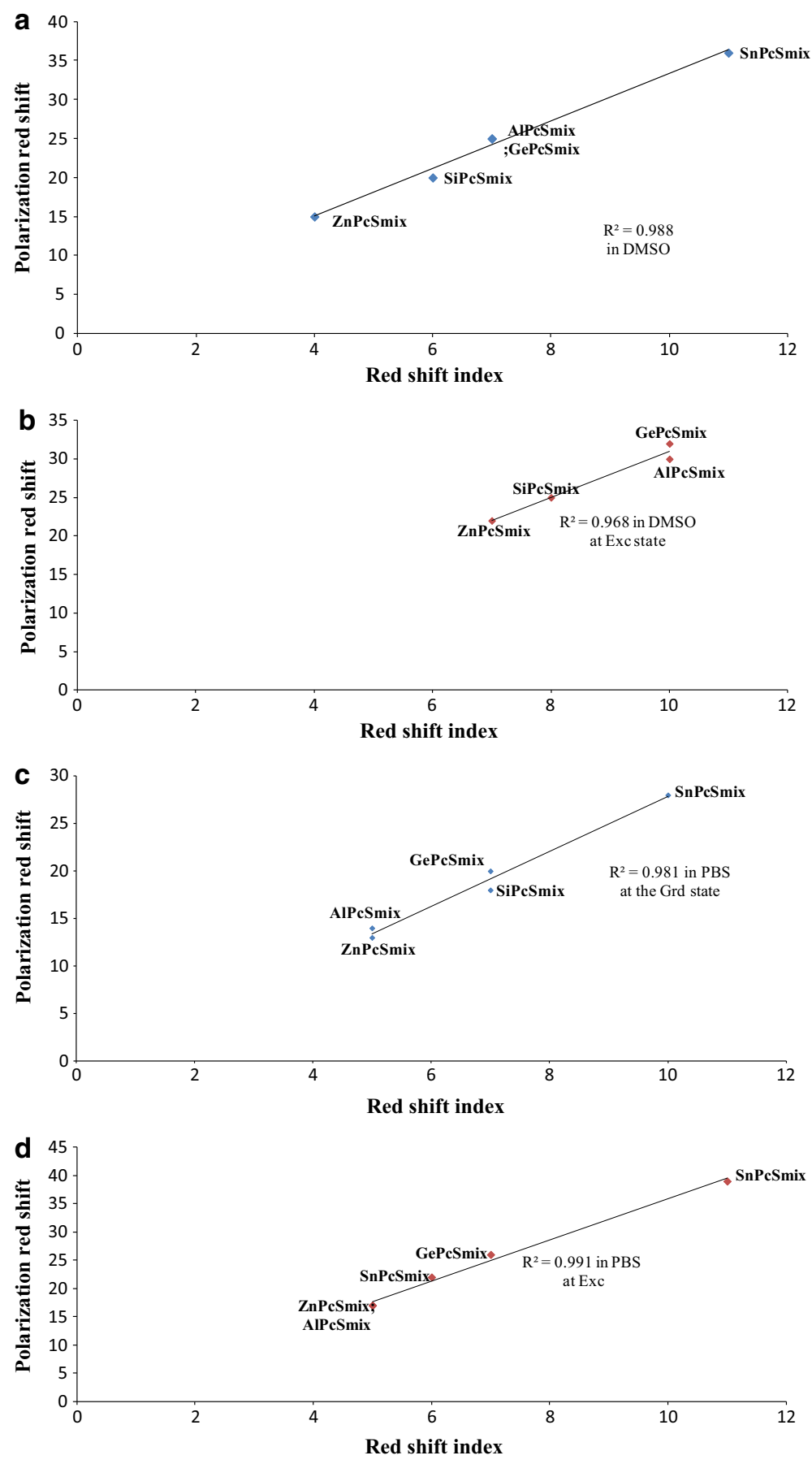

Fig. 3 a Polarization red shift versus red shift idex, $R_{s} I$, at the ground state in DMSO. b Polarization red shift versus $R_{s} I$ at the excited state in DMSO. c Polarization red shift versus $R_{s} I$ at the ground state in DMSO. d Polarization red shift versus $R_{s} I$ at the excited state 

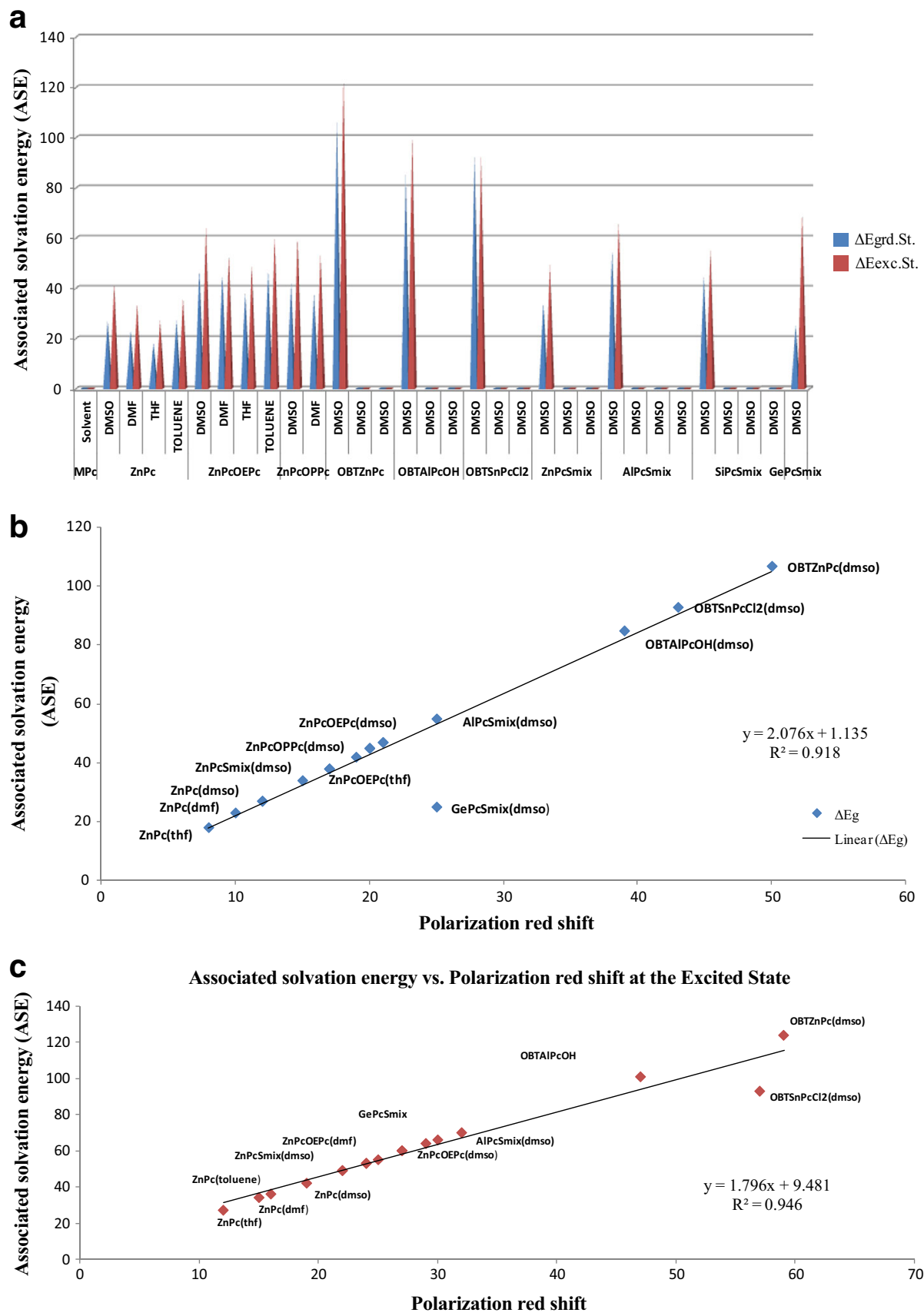

Fig. 4 a Associated solvation energy of MPc derivative. b Associated solvation energy versus polarization red shift at the ground state. c Associated solvation energy versus polarization red shift at the excited state 
Fig. 5 MPc ranking based on statistical mean performance of solvatochromic parameters

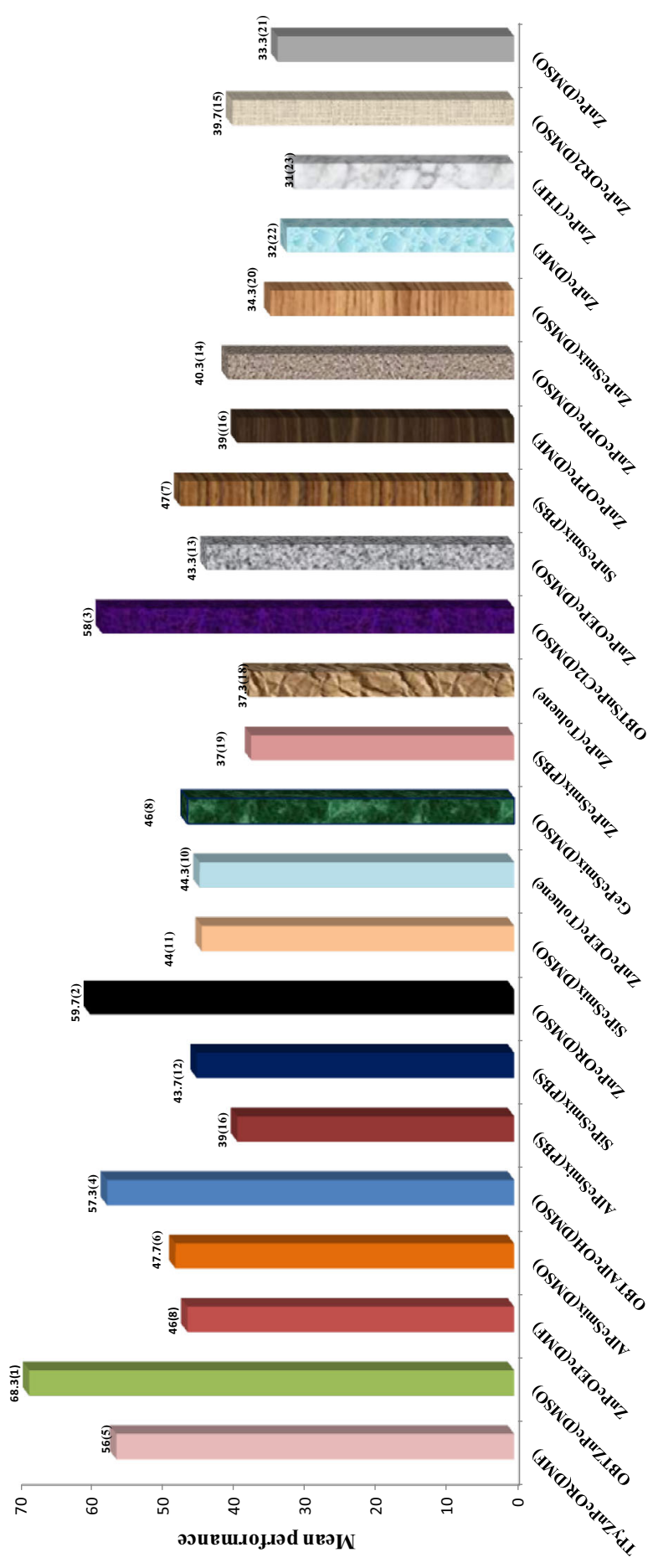

算 Springer 
activity between the solute-solvent interactions in the ground state than the excited state. The coordinating nature of DMF is more polar in the ground state and thus brings about the increased polarization red shift seen in the ground state and the reduced polarization red shift seen in the excited state.

The solvation trend of both positive and negative solvent effects is illustrated by the correlation plots of the polarization red-shift versus the red-shift index of MPcs with sulphonated moieties at the ground and excited state shown in Fig. 3a-d. Figure 3a, b show the positivesolvent effect at the ground and excited state in DMSO, respectively. At for the ground state, the solvation trend observed shows the following order: SnPcSmix $>$ GePcSmix $>$ AlPcSmix $>$ SiPcSmix $>$ ZnPcSmix, which is the same trend observed at the excited state. This therefore means that the the positive solvent effect is stabilized in DMSO as shown by the $R^{2}$ values to be 0.988 and 0.968 for the ground and excited states, respectively. A similar trend was observed for the negative solvent effect in PBS in Fig. 3c, d, where the series are stabilized along the solvation trend in this order at both the ground and excited state: SnPcSmix $>$ GePcSmix, SiPcSmix > AlPcSmix, ZnPcSmix. The $R^{2}$ values for the respective ground and excited states in the correlation graphs gave 0.981 and 0.991 , respectively. This study thus indicates that solvation process is affected by the nature of solute-solvent polarities of MPcSmix in DMSO and PBS, described in this study as positive and negative solvent effects.

Table 2 shows the parameters used for entering the values for the associated solvation energy (ASE) of CS-MPc, which is an important concept derived from the red shift index. Table 2 thus shows that the ASE values correlate well with all the parameters. In Fig. 4a, the ASE chart shows the ASE variation across the different solvents with reference to DMSO. The result shows the ASE of the excited state to be greater than ASE at the ground state, which is to be expected as the polarization red-shift of all positive solvent effect trends are expected to be higher in the excited state than the ground state. The ASE trend in the different solvents shows the OBTMPc group to exhibit greater solvation than the respective $\mathrm{CS}-\mathrm{MPc}$, where $\mathrm{OBT}(\mathrm{Zn}>\mathrm{Al}>\mathrm{Sn})$ for both the ground and the excited states. Figure $4 \mathrm{a}$ also shows that ASE at the excited state is greater than at the ground state across the different solvent media for the different CS-MPcs. Figures $4 b$, c show the correlation plot of the ASE variation versus the polarization red-shift at both the ground and the excited states with $R^{2}$ values at 0.918 and 0.946 , respectively.

Table 3 shows the parameters used for entering the data for CS-MPc ranking and thus includes the red shift index $\left(R_{S} I\right)$, polarizability efficiency and the associated solvation energy (ASE). To achieve the ranking, a statistical mean performance of the $R_{s} I$, polarizability efficiency and ASE were taken, from which the mean along with the standard deviation from the mean were calculated.

Figure 5 therefore shows the chart of MPc ranking based on the statistical mean performance of the solvatochromic parameters explained in Table 3, with OBTZnPc (DMSO) ranked as 1 , followed by $\mathrm{ZnPcOR}$ (DMSO) and the last, $\mathrm{ZnPc}$ (THF). The stastical progression of the ranking shows a true reflection of the mean performance that is dependent on the nature of chromophore substituent (CS) attached to the MPc periphery or 'handles' and the interactions of solute-solvent at the ground and excited states.

\section{Conclusions}

The red-shift index has been derived to study the behaviors of CS-MPc polarities in different solvent media in both the ground and the excited states. The $R_{S} I$ and ASE are parameters developed to study the underlying solute-solvent interactions. We have been 
able to demonstrate that the spectral approach provides a good empirical way of examining the macroscopic properties as shown by the data obtained in Tables 1,2 and 3. Therefore within the defined parameters, the mathematical formulation for $R_{S} I$ shows that it is reproducible and can be used to tailor CS-MPcs at the macroscopic level. The ASE concept derived from $R_{s} I$ also shows that the associated solvation energy-solvent interactions can be determined at the macroscopic level. Overall, the totality of the $R_{s} I$, polarizability efficiency and ASE shows a good empirical way of evaluating the ranking of CS moieties at both the ground and the excited state in the respective solvents. This study shows a new method for both studying the spectroscopic trends of MPcs and the behaviors of chromophore substituents in various solvent media at the macroscopic level.

Acknowledgments This study was supported by the Royal Institute of Technology, KTH, Stockholm, Sweden and scholarship support was provided by the Swedish Institute and KTH.

\section{References}

1. Hagan, R., Bieringer, T.: Photoaddressable polymers for optical data storage. Adv. Mater. 13, 1805-1810 (2001)

2. Oni, J., Westbroek, P., Nyokong, T.: Voltammetric detection of vitamin B1 at carbon paste electrodes and its detection in tablets. Electroanalysis 14, 1165-1168 (2002)

3. Vemkatram, N., Narayana, R.D., Giribabu, L., Rao, V.S.: Nonlinear optical and optical limiting studies of alkoxy phthalocyanine in solutions studied at $532 \mathrm{~nm}$ with nanosecond pulse excitation. Appl. Phys. B 91, 149-156 (2008)

4. Beeby, A., FitzGerald, S., Stanley, C.F.: A Photophysical study of protonated (tetra-tert-butyl phthalocyaninato) zinc. J. Chem. Soc. Perkin Trans. 2, 1978-1982 (2001)

5. Akpe, V., Vernet, M.C., Obirai, J.C., Brismar, H.: Understanding the photochemical pathway of in vitro target delivery of aluminium phthalocyanine: a mechanistic approach using radical reaction chemistry. ChemPlusChem 79, 671-679 (2014)

6. Rosenkranza, A.A., Jans, D.A., Soboley, S.A.: Targeted intracellular delivery of photosensitizers to enhance photodynamic efficiency. Immunol. Cell Biol. 78, 452-464 (2000)

7. Kadish, K.M., Smith, K.M., Guilard, R.: The Porphyrin Handbook: Phthalocyanines: Spectroscopic and Electrochemical Characterization, vol. 16, pp. 4, 15-4 35. Academic Press, San Diego (2003)

8. Wei, S., Deyin, H., Li, L., Qinghua, M.: Synthesis and properties of some novel soluble metallopthalocyanines containing the 3-trifluoromethylphenoxy moiety. Dyes Pigment 56, 1-6 (2003)

9. Dini, D., Guo, Y.Y., Michael, H.: Perfluorinated phthalocyanines for optical limiting: evidence for the direct correlation between substituent electron withdrawing character and the nonlinear optical effect. J. Chem. Phys. 119, 4857-4864 (2003)

10. Bayır, Z.A.: Synthesis and characterization of novel soluble octa-cationic phthalocyanine. Dyes Pigment 65, 235-242 (2005)

11. Reichardt, C.: Solvent and Solvent Effects in Organic Chemistry, 2nd edn, pp. 359-384. VCH, Weinheim (1998)

12. Nyokong, T.: Effects of substituents on the photochemical and photophysical properties of main group metal phthalocyanines. Coord. Chem. Rev. 251, 1707-1722 (2007)

13. Bayliss, N.S., McRae, E.G.: Solvent effects in organic spectra: dipole forces and the Franck-Condon principle. J. Phys. Chem. 58, 1002-1006 (1954)

14. Bayliss, N.S., McRae, E.G.: Solvent effects in the spectra of acetone, crotonaldehyde, nitromethane and nitrobenzene. J. Phys. Chem. 58, 1006-1011 (1954)

15. Amos, A.T., Burrows, B.L.: Solvent-effects on electronic spectra and excited-state dipole moments and polarizabilities. Adv. Quantum Chem. 7, 289-313 (1973)

16. Orozco, M., Luque, F.: Theoretical methods for the description of the solvent effect in biomolecular system. Chem. Rev. 100, 4187-4225 (2000)

17. Ogunsipe, A., Maree, D., Nyokong, T.: Solvent effects on the photophysical and fluorescence properties of zinc phthalocyanine derivatives. J. Mol. Struct. 650, 131-140 (2003)

18. Akpe, V., Brismar, H., Nyokong, T., Osadebe, P.O.: Photophysical and photochemical parameters of Octakis(benzylthio) phthalocyaninato zinc, aluminium and tin: red shift index concept in solvent effect on the ground state absorption of zinc phthalocyanine derivatives. J. Mol. Struct. 984, 1-14 (2010) 
19. Freed, B.K., Biesecker, J., Middleton, W.J.: Spectral polarity index: a new method for determining the relative polarity of solvents. J. Fluor. Chem. 48, 63-75 (1990)

20. Durmus, M., Nyokong, T.: Synthesis and solvent effects on the electronic absorption and fluorescence spectral properties of substituted zinc phthalocyanines. Polyhedron 26, 2767-2776 (2007)

21. Chidawanyika, W., Ogunsipe, A., Nyokong, T.: Synthesis and photophysics of new phthalocyanine derivatives of zinc, cadmium and mercury. New J. Chem. 31, 377-384 (2007)

22. Ogunsipe, A., Nyokong, T.: Photophysical consequences of bovine serum albumin binding to nontransition metal phthalocyanine sulfonates. Photochem. Photobiol. Sci. 4, 510-516 (2005)

23. Ogunsipe, A., Nyokong, T.: Photophysical and photochemical studies of sulphonated non-transition metal phthalocyanines in aqueous and non-aqueous media. J. Photochem. Photobiol. A 173, 211-220 (2005) 